\title{
Investigation and Analysis of Continuing Nursing Education Status and Needs of In-Service Nurses in Tibet
}

\author{
Haihua Zou1, Lihong Liu' ${ }^{2}$, Lijuan Dong2, Weiju Chen ${ }^{*}$ \\ ${ }^{1}$ Nursing Department of the First Affiliated Hospital of Jinan University, Guangzhou, China \\ ${ }^{2}$ Linzhi People's Hospital, Linzhi, China \\ Email: *313761095@qq.com
}

How to cite this paper: Zou, H.H., Liu, L.H., Dong, L.J. and Chen, W.J. (2020) Investigation and Analysis of Continuing Nursing Education Status and Needs of In-Service Nurses in Tibet. Open Journal of Internal Medicine, 10, 223-231.

https://doi.org/10.4236/ojim.2020.102023

Received: April 30, 2020

Accepted: May 25, 2020

Published: May 28, 2020

Copyright ( $\odot 2020$ by author(s) and Scientific Research Publishing Inc. This work is licensed under the Creative Commons Attribution International License (CC BY 4.0).

http://creativecommons.org/licenses/by/4.0/

\begin{abstract}
Objective: To investigate and analyze the current status of continuing nursing education (CNE) of in-service nurses in Tibet and their demand for continuing education. Methods: 663 active nurses in Tibet were taken as the research objects, and the self-developed questionnaire was used to investigate the current status and needs of CNE, and the correlation between the CNE needs of active nurses and their personal conditions was analyzed. Results: This study shows that $65.61 \%$ (435/663) of active nurses have not participated in any CNE, and $91.86 \%(609 / 663)$ of active nurses believe that they need to participate in CNE. The problems faced by active nurses in participating in CNE include funding restrictions, time limitation and ability limitation. Logistic stepwise regression analysis reveals that the age of active nurses and working years of nurses are negatively correlated with the funding restrictions for participating in CNE $(\mathrm{P}<0.05)$. It is relatively more difficult for young nurses with less working experience to obtain funding for participating in CNE. Moreover, there is a negative correlation between age, educational level and the ability of nurses to participate in CNE $(\mathrm{P}<0.05)$. Younger nurses with lower educational level are more difficult to participate in CNE due to personal ability. There is a positive correlation between the length of working experience and the time limitation faced to participate in CNE $(\mathrm{P}<0.05)$. Senior nurses are too busy with their work to participate in CNE due to limited time. Conclusion: The problems faced by active nurses in Tibet in participating in CNE come from funding, time and ability. Young nurses with limited working experience cannot participate in CNE resulting from limited ability and funds; while senior nurses are mainly restricted by time. It is recommended that hospitals and nursing management in Tibet should solve these problems faced by nurses in participating in CNE by implementing a
\end{abstract}


flexible scheduling system, and providing funding or implementing an incentive system, to enhance the confidence of active nurses in participating in CNE.

\section{Keywords}

Tibet, In-Service Nurses, Continuing Education, Current Situation Analysis, Facing Problems, Solutions

\section{Introduction}

Continuing nursing education (CNE) refers to the content of lifelong nursing education that nurses receive after completing standardized professional training [1] [2]. The emergence of CNE can promote both the personal development of in-service nurses and the national development of medical clinical nursing level in China [3]. In recent years, many medical institutions in various regions of China have carried out CNE activities in various forms, and CNE has been effectively promoted in many places [4]. However, when aiding in Tibet we found that the promotion of CNE in Tibet is facing many challenges. Although the active nurses in Tibet have a high demand for CNE, the nurses have an insufficient understanding of CNE and few opportunities for participating. Tibet is located in the southwestern frontier of China, which is a special geographical location. However, the economy of Tibet is not well developed and is relatively backward compared to other provinces, and medical education resources are relatively scarce. In this regard, it is necessary to fully understand the current status of CNE implementation in Tibet from the perspective of in-service nurses and provide a reference basis for exploring the formulation of CNE education channels and related policies suitable for in-service nurses in Tibet [5].

\section{Objects and Methods}

\subsection{Research Object}

The research survey was carried out from January to March 2020. The study selected 24 hospitals in Tibet (6 first-class A level hospitals, 10 second-class A level hospitals, and 8 second-class $\mathrm{B}$ level hospitals) through a stratified cluster random sampling survey.

The principles of sample size calculation include: 1) To ensure the quality of the survey, the number of nurses participating in the survey in each hospital is $\geq 20$; 2 Calculation formula conforming to sample size: $n=\mu_{\alpha / 2}^{2} \pi(1-\pi) / \delta^{2}$; $n_{c}=n /(1+n / N), \pi$ takes Hangzhou norm, $\pi=0.591, \delta=0.05, \alpha=0.05, \mu \alpha / 2=$ 1.96 , Consider $20 \%$ sample loss, $N \approx 377$, corrected $n_{c} \approx 314$, That is, Included sample quantity $\geq 314$ people; A total of 663 registered nurses received anonymous questionnaire. Inclusion criteria for the nurse: a) Active nurse should obtain the People's Republic of China Nurse Practitioner Certificate; b) The 
nurse should have at least 1 year working experience; c) Currently the nurse is in clinical or nursing management positions. Exclusion criteria: exclude nurses who were not in the position during the questionnaire; exclude nurses who are unwilling or unable to participate in this survey due to other factors. The nurses involved in the study had informed consent to the content of the study, and the study was approved by the ethics of regional hospitals, and the survey content met the requirements of medical ethics.

\subsection{Investigation Tools}

The research adopted a questionnaire survey method. Following by reviewing literatures on "CNE, active nurses and Tibet" [6] [7] [8], the members of the research team developed the Questionnaire Continuing Nursing Education Status and Needs for Active Nurses in Tibet, which is hereinafter referred as the questionnaire, based on professional opinions from experts in Guangdong Province and Tibet. The questionnaire mainly includes general nurse information (10 items), nurse CNE status (10 items) and nurse needs for CNE learning (5 items). Before the formal investigation, 20 subjects were randomly selected to test the reliability of the questionnaire, and the internal consistency coefficient Cronbach's $\alpha$ is 0.927 , Cronbach's $\alpha$ of current situation of CNE education for nurses is 0.953 , and Cronbach's $\alpha$ of CNE learning needs of nurses is 0.916 .

\subsection{Method}

An electronic link of the questionnaire was sent to the respondents, and each mobile terminal is restricted to fill in the questionnaire only once to avoid repeating; the questionnaire was withdrawn within 2 days from the issuing date, and the questionnaire with consistent answers and logical contradictions was excluded. A total of 678 questionnaires were distributed in the study, and 663 valid questionnaires were received with an effective recovery rate of the questionnaire at $97.78 \%$.

\subsection{Statistical Processing}

SPSS statistical software (version 19.0) was used to analyze and process the data. Among them, the count data was described in the form of $\mathrm{N}$ (\%), and the non-normal distribution data was described in the form of interquartile range-median form. Multivariate logistic regression analysis was used analyze the relation between the continuing education needs of active nurses and their personal conditions. $\mathrm{P}<0.05$ indicates that the comparison is of statistical significance.

\section{Results}

\subsection{General Information of the Respondents}

The survey included 663 respondents. The general information of involved active nurses is shown in Table 1. 
Table 1. Analysis of the general information of the respondents $(\mathrm{N}=663)$.

\begin{tabular}{|c|c|c|}
\hline Item & Classification & Count (Proportion) \\
\hline \multirow[t]{2}{*}{ Sex } & Male & 27 \\
\hline & Female & 636 \\
\hline \multirow[t]{3}{*}{ Ethnic } & Han & $204(30.77 \%)$ \\
\hline & Tibetan & $399(60.18 \%)$ \\
\hline & other & $60(9.05 \%)$ \\
\hline Age (a) & - & $27(19,52)$ \\
\hline Working years (a) & - & $7.0(1.0,30.0)$ \\
\hline \multirow[t]{6}{*}{ Current position } & Director/Deputy Director & $27(4.07 \%)$ \\
\hline & Head nurse & $81(12.22 \%)$ \\
\hline & Specialist nurse & $18(2.71 \%)$ \\
\hline & Nursing team leader & $57(8.6 \%)$ \\
\hline & Teaching nurse & $9(1.36 \%)$ \\
\hline & Nurse & $471(71.04 \%)$ \\
\hline \multirow[t]{4}{*}{ Educational background } & Master's degree or above & $0(0 \%)$ \\
\hline & Bachelor's degree & $294(44.34 \%)$ \\
\hline & College & $336(50.68 \%)$ \\
\hline & Technical secondary school & $33(4.98 \%)$ \\
\hline \multirow[t]{5}{*}{ Job Title } & Chief Nurse & $0(0 \%)$ \\
\hline & Deputy Chief Nurse & $2(0.3 \%)$ \\
\hline & Supervisor in charge & $46(6.94 \%)$ \\
\hline & Nurse (female) & $273(41.18 \%)$ \\
\hline & Nurse (male) & $471(71.04 \%)$ \\
\hline
\end{tabular}

\subsection{Analysis of Current Status and Challenges of Active Nurses Participating in CNE}

It suggests that $65.61 \%(435 / 663)$ of active nurses have not participated in any CNE, but $91.86 \%(609 / 663)$ of them believe that they need to participate in CNE. The challenges faced by these active nurses participating in CNE include: attending CNE requires self-financing, and nurses have financial difficulties (fund restrictions); training time restrictions (time restrictions); nursing scheduling is difficult, and nursing personnel is insufficient to limited learning time (time restrictions); nurses have insufficient capability to participate in CNE learning and are lack of confidence (ability limitation), as shown in Table 2.

\subsection{Relevance Analysis of the Challenges Faced by Nurses Participating in CNE and Their Personal Conditions}

In the study, the personal data of the nurses were used as independent variables, and the difficulties in continuing education (fund limitation, time limitation, 
ability limitation) were used as dependent variables in turn. Multivariate logistic regression analysis showed that the age, working years and academic level were correlated with the challenges for the nurses participating in $\mathrm{CNE}(\mathrm{P}<0.05)$.

The age and working years of nurses are negatively correlated to the funding restrictions for participating in $\mathrm{CNE}(\mathrm{P}<0.05)$. It is relatively more difficult for young nurses with limited working experience to obtain funds for participating in CNE. The age and educational level of nurses have a negative correlation with the ability limitations of participating in CNE $(\mathrm{P}<0.05)$, and younger nurses with lower educational level are more difficult to participate in CNE due to personal capabilities. the length of work experience is positively correlated with the time limitation for participating in CNE $(\mathrm{P}<0.05)$, senior nurses are constrained to participate in CNE due to their busy schedule, as shown in Table 3.

\section{Discussion}

\subsection{Current Status and Challenges of Active Nurses Participating in CNE Learning}

This study shows that $65.61 \%$ of active nurses have not participated in any CNE, which is lower than other regions. A CNE survey of nurses in medical institutions in Taiyuan City shows that [9] the proportion of nurses participating in CNE projects in $t$ Taiyuan City is $80.02 \%$ (2130/2662); An analysis of the status of CNE for 244 active nurses in Zhejiang Province shows that [10], more than $96 \%$ of nurses have participated in CNE. Thus, the survey results suggest that CNE for active nurses in Tibet needs to be further developed.

Table 2. Current status and problem analysis of nurses participating in CNE learning (N $=663$ ).

\begin{tabular}{|c|c|c|}
\hline Challenges & $\mathbf{N}$ & Percentage \\
\hline $\begin{array}{l}\text { Participation in training requires self-finance, and nurses have financial } \\
\text { difficulties }\end{array}$ & 336 & $50.68 \%$ \\
\hline Training time limit & 435 & $65.61 \%$ \\
\hline $\begin{array}{l}\text { Nursing scheduling is difficult, insufficient nursing manpower restricts } \\
\text { study time }\end{array}$ & 528 & $79.64 \%$ \\
\hline Limited learning capability and lack of confidence to participate in CNE & 228 & $34.39 \%$ \\
\hline
\end{tabular}

Table 3. Correlation analysis between the CNE needs of active nurses in Tibet and their personal conditions.

\begin{tabular}{cccccc}
\hline Independent variable & dependent variable & B & SE & $\boldsymbol{r}$ & P \\
\hline Nurse's age & Funding limit & -0.812 & 0.142 & -0.633 & $<0.05$ \\
Working years & Capability limit & -0.827 & 0.149 & -0.658 & $<0.05$ \\
& Funding limit & -0.835 & 0.151 & -0.672 & $<0.05$ \\
Educational level & Time limit & 0.637 & 0.179 & 0.526 & $<0.05$ \\
\hline
\end{tabular}


91.86\% of active nurses believe that they need to participate in CNE. The survey shows that active nurses in Tibet generally have the need to participate in CNE training. Lu et al. [11] pointed out that the challenges faced in the CNE of in-service nurses include both the subjective and objective reasons of the nurses themselves as well as time constraints. Some other problems include that the content of CNE is not systematic and novel enough, or the forms and methods cannot meet the learning needs of the nurses. The survey in this study suggests that the challenges faced by in-service nurses participating in CNE are: attending training requires their own funding; the nurses have financial difficulties; training time restrictions; difficult nursing scheduling, insufficient nursing manpower restricts learning time; nurses have insufficient capability to participate CNE and lack of confidence. Therefore, funding, time, and capacity limitations are the main issues challenge the current working nurse in Tibet to participate in CNE. Multivariate logistic regression analysis showed that the age, working years and academic level were correlated with the challenges for the nurses participating in CNE. The age and working years of nurses are negatively related to the funding restrictions for participating in $\mathrm{CNE}$, indicating that young nurses with limited working experience had much more difficulties to obtain funds for participating in CNE. The age and educational level of nurses is negatively correlated with the capacity limitations of nurses participating in CNE, suggesting that younger nurses with lower educational levels are more difficult to participate in CNE due to personal capabilities. The length of work experience is positively correlated with the time limitation for nurses participating in $\mathrm{CNE}$, indicating that senior nurses intending to participate in CNE are subject to time constraints. Ni et al. [12] studied the problems existing in the participation of nurses in CNE and found that the main factors affecting the participation of in-service nurses in CNE included busy work, high cost, limited region and disallowed family situation. The results of this study are similar to those of the study; they are both suggesting that nurses' ability and time are the factors that affect their participation in CNE. In the study, only in-service nurses in Tibet were investigated, excluding the influence of regional restriction, but the results also suggested that the age of nurses was also the main factor limiting their participation in CNE.

\subsection{Countermeasures to Solve the Problem of In-Service Nurses Participating in CNE}

The hospital and nursing management should provide increasing support for $\mathrm{CNE}$ and strive to explore and improve the strategy of nurses to participate in CNE. 1) In view of the time limitations faced by in-service nurses participating in $\mathrm{CNE}$, hospitals and nursing management should change their minds and arrange nurses in a reasonable and flexible manner. For nurses participating in $\mathrm{CNE}$, reasonable arrangements should be made to alleviate the time conflict between nurse working and studying. Wang et al. [12] found that the implementation of flexible scheduling can improve the satisfaction of clinical nurses in their nursing work. The study shows that flexible scheduling improves nurses' profes- 
sional satisfaction, eliminates or reduces overtime, and promotes the cooperation of the nurses and patients, and nurses can get more adequate rest in their free time. In addition to effective exercise at work, young nurses also have enough spare time to improve their own nursing level through CNE. 2) In view of the funding limitations faced by in-service nurses participating in CNE, it is recommended that hospitals in the Tibetan region consider adopting financial assistance from hospitals or granting financial incentives based on the actual performance of nurses to promote them to participate CNE. Agnew A J et al. [13] showed that learning incentives can help medical students develop self-direction and self-regulation and other learning behaviors, suggesting that the incentive mechanism has positive significance in promoting nurses to participate in CNE. Therefore, it is essential to develop effective mechanisms to encourage nurses in emergency departments to consciously participate in CNE. The research suggests that an effective incentive mechanism has important value in improving the effectiveness of nurses' CNE. 3) In view of the personal capability limitations faced by in-service nurses to participate in CNE, the hospital nursing management department needs to be well analyzing the characteristics of nurses at different stages of learning and formulating a personalized continuing education plan. The goal of CNE is to improve academic qualifications, nursing skills and nursing research capabilities of participated nurses. To achieve this goal, it is necessary to improve the learning efficiency of the nurses participating in CNE by adopting scientific, systematic, diversified and flexible methods [14] [15]. Gu et al. [16] believe that in the process of arranging the fundamental professional skills of nurses, the nursing management department should not only enable nurses to learn new knowledge and skills in medicine and nursing according to the laws of medical development, but also arrange training in social humanities and psychology, which could increase the confidence of nurses in actively participating in CNE.

\subsection{Limitations of Research}

This study only analyzes the current situation and objective problems of in-service nurses' participation in CNE learning in Tibet. In view of the needs of learning approach, learning content and training objectives of in-service nurses in Tibet, further research is needed. At present, there are many ways for nurses to participate in CNE, including out of hospital further education, in hospital training, short-term out of hospital training courses, high-level academic education, continuing education training courses or seminars, and distance education. It is one of the limitations of this study that there is no in-depth discussion on what kind of education way should be provided for in-service nurses in Tibet; At the same time, the current learning content of in-service nurses for CNE mainly includes improving basic nursing theory and skills, progress and theory of specialized nursing, new nursing technology, hospital infection control and occupational protection, nursing management, nursing education, interpersonal communica- 
tion, psychology, nursing research and nursing laws and regulations. It is also a key research direction to further standardize the CNE training of in-service nurses in Tibet. In the follow-up study, the author will carry out further research on the CNE learning path, learning content needs and the training objectives of in-service nurses in Tibet in the future.

\section{Conclusion}

The training of grassroots nurses is a key measure to strengthen grassroots health services in our country and effectively promote the implementation of the graded diagnosis and treatment system. Tibet has a special geographical location with inconvenient transportation resulting in difficulties for local patients in seeking medical treatment in other areas. Therefore, it is necessary and of positive significance to effectively improve the technical ability of in-service nurses in Tibet, accelerate the cultivation of regional nursing talents, and improve the quality of medical and health services in Tibet and accelerate the development of medical treatment. This research survey shows that more than half of the working nurses in Tibet believe that there are certain pressures and challenges in participating in the CNE. Their main problems are funding, time and capability. Specifically, young nurses with limited working experience are affected by restrictions on capability and funding, while senior nurses are mainly limited by time. Our study further reveals that to carry out and implement CNE for the active nurses in Tibet in the future, the local government and hospitals should take many aspects into consideration, such as organization, medical institutions, individual nurse and nurse's family conditions. It is recommended that hospitals and nursing management in Tibet should resolve these challenges through the implementation of flexible scheduling systems, assistance from hospital funding or the implementation of incentive systems, and the enhancement of confidence of nurses participating in CNE. This would provide a practical path for the personalized career development of in-service nurses in Tibet.

\section{Conflicts of Interest}

The authors declare no conflicts of interest regarding the publication of this paper.

\section{References}

[1] He, K.Y., Ye, C.Y. and Tan, X.X. (2019) Analysis of the Application Value of Different Continuing Education Modes in Improving the Nursing Ability of Nursing Staff. Journal of Rare Diseases, 26, 103-105.

[2] Dunker, S., Kimberly, M., et al. (2018) Live Continuing Education Program for Adjunct Clinical Nursing Faculty, 39, 16. https://doi.org/10.1097/01.NEP.0000000000000248

[3] Cheng, S.S. and Ma, X.Q. (2019) The Qualitative Study on the Cognition and Experience of Continuing Education for Nurses. Higher Medical Education in China, 8, 33-34. 
[4] Wang, Q., Zhou, H.M. and Chen, J. (2019) Investigation and Analysis on the Status Quo, Learning Attitude and Influencing Factors of Nursing Staff in Hospital Continuing Education and Training. Nursing Practice and Research, 16, 22-24.

[5] Dang, Y. and Jiao, G.M. (2017) Discussion on the Application of Constructivism Learning Theory in the Teaching of Nursing Continuing Education. Journal of Inner Mongolia Medical University, 39, 209-212.

[6] Dyck, M.J. and Kim, M.J. (2018) Continuing Education Preferences, Facilitators, and Barriers for Nursing Home Nurses. Journal of Continuing Education in Nursing, 49, 26. https://doi.org/10.3928/00220124-20180102-07

[7] Wang, X.L. and Song, L.J. (2019) The Evaluation of the Practical Effect of Nursing Education Procedure in Nurse Level Training. Guangdong Vocational and Technical Education and Research, 2, 196-200.

[8] Park, Y.-S., Kim, J.-A., et al. (2018) Application of Team-Based Learning as a Teaching Method in Nursing Education. Journal of Medical Imaging \& Health Informatics, 8, 439-443. https://doi.org/10.1166/jmihi.2018.2327

[9] Gao, X.L. (2019) Investigation and Analysis on the Status Quo of Continuing Education of Nurses in Medical Institutions in Taiyuan. Chinese Medicine and Clinic, 19, 566-568.

[10] Sun, X.Y., Lu, J.F., Jiang, Y.L., et al. (2010) Analysis on the Current Situation of Continuing Education of In-Service Nurses and Its Countermeasures. Journal of Nursing Training, 25, 407-409.

[11] Lu, A.P., Yang, X., Zhou, K.X., et al. (2002) Analysis of the Problems in the Operation of Continuing Nursing Education. Chinese Journal of Nursing, 8, 43-45.

[12] Wang, X.L., Yang, S.Y., Zhu, Z.L., et al. (2013) Effect of Flexible Schedule on Job Satisfaction of Nurses. Nursing Practice and Research, 10, 107-108.

[13] Agnew, A.J. and O’Kane, C.J. (2011) Addressing the Hiatus of Learning Incentives for Prevocational Doctors: Continuing Medical Education Points for Interns. Medical Journal of Australia, 194, 349. https://doi.org/10.5694/j.1326-5377.2011.tb03004.x

[14] Yang, Q.H. (2018) Observation on the Application Effect of Wechat Platform Management in Continuing Education of Nursing Staff. Nursing Practice and Research, 15, 140-142.

[15] Cronin, S.N., Berger, J., Nelson, D., et al. (2019) Crossing Boundaries to Advance Nursing Research Education. Journal for Nurses in Professional Development, 35, 1-5. https://doi.org/10.1097/NND.0000000000000523

[16] Gu, X.Y. (2014) Investigation and Analysis on the Needs of Nurses of Different Working Age for Continuing Nursing Education. Chinese Health Industry, 11, 53-54+56. 